\title{
Use of a virtual reality car-driving stressor in cardiovascular reactivity research
}

\author{
J. RICK TURNER, FRANK A. TREIBER, HARRY DAVIS, \\ JOSEPH RECTANWALD, WALTER PIPKIN, and WILLIAM B. STRONG \\ Medical College of Georgia, Augusta, Georgia
}

\begin{abstract}
Eighteen subjects (11 males, 7 females) completed a virtual reality car-driving stressor on two occasions several weeks apart. Immediately before and throughout task performance, blood pressure, cardiac output, and total peripheral resistance were assessed. Reactivity scores were calculated for each parameter for each subject as the arithmetic difference between task level and baseline level. The task elicited considerable hemodynamic activation on each occasion of testing, as well as high levels of self-reported task realism, engagement, excitement, and nervousness. Correlation analyses of both absolute and reactivity scores revealed evidence of test-retest reliability. Males were found to exhibit greater absolute levels of and greater increases in systolic blood pressure. The development of tasks suitable for inclusion in a battery of behavioral stressors, responses to which may help identify those at risk for later disease, is of considerable interest in cardiovascular behavioral medicine. The present findings suggest that the virtual reality car-driving task may be useful in this context.
\end{abstract}

Investigation of individual differences in cardiovascular responses to stress has, in recent years, attracted significant attention in light of hypotheses linking chronic environmental stress-induced cardiovascular adjustments to later cardiovascular pathophysiology (Manuck, 1994; Matthews et al., 1986; Obrist, 1981). Stress-testing protocols employed in cardiovascular behavioral medicine have utilized a wide variety of tasks to elicit stress in the laboratory and hence to examine individual differences in cardiovascular responsivity during various challenges. Some of these tasks have been physical in nature and have included isometric (i.e., handgrip dynamometer) and dynamic (e.g., bicycle ergometry, treadmill) exercise and variations of the cold pressor task that involve placement of a limb in iced water or, alternatively, placement of a bag of iced water on the forehead (see, e.g., Durel et al., 1993; Matthews et al., 1986). Longitudinal studies have yielded inconsistent results as to the value of cardiovascular reactivity to these physical stressors as an independent predictor of future cardiovascular disease (for a review, see Manuck, 1994).

One rationale for the lack of consistent findings regarding the predictive value of cardiovascular reactivity to such physical stressors is that these tasks are not representative of the types of environmental stress most individuals encounter on a daily basis. As a result, a second category of "psychological" stressors has gradually emerged for use in the laboratory setting. These stressors are deliberately constructed so that motor responses are minimal, often consisting of moving one or two fingers to depress response keys. Psychological stressors can be operationally defined

This work was supported by NIH Grants 41781 and 35703 . Correspondence should be addressed to F. A. Treiber, Medical College of Georgia, Georgia Prevention Institute, HS-1640, Augusta, GA 30912-3710 (e-mail: ftreiber@mcg.edu). as those that demand mental effort and active engagement in the task in order to achieve reasonable task completion and that, as noted above, require minimal physical exertion. Tasks falling into this category include reaction time tasks (Obrist, 1976), mental arithmetic tasks (Brod, Fencl, Hejl, \& Jirka, 1959), speech tasks (Saab, Matthews, Stoney, \& McDonald, 1989), and video games (Turner, Carroll, \& Courtney, 1983). Further, a recent advancement in assessment of cardiovascular reactivity has been the use of a diverse battery of stressors (see Kamarck et al., 1992). Responses to the tasks contained in such a battery can be aggregated, thereby producing a more representative assessment of an individual's responsivity than would be given by a single task. However, most of these stressors have not been reflective of commonly encountered daily events. Given that cardiovascular disease has its origins in childhood (Matthews et al., 1986), it is also important for such tasks to be applicable to a wide range of ages. The present report offers a new task that addresses this need.

Recent advances in virtual environment technology have facilitated the development of extremely realistic, highly immersive, real-time simulations of various reallife situations, some of which may be particularly useful in cardiovascular reactivity research (Moshell \& DunnRoberts, 1993). One of these is the car-driving simulation task; this task can in some ways be regarded as an improved version of video games, which have also been used successfully in this research domain. The car-driving simulation contains the elements of uncertainty and symbolic avoidance (of crashes) that help generate the necessary levels of continuous task involvement and vigilance that are required for psychological tasks to affect the hemodynamic processes of interest.

The car-driving task has advantages for use with both adolescents and adults. It has been argued that tasks de- 
ployed in laboratory investigations should reflect the situations individuals encounter in everyday life. Driving is a common experience for many adolescents and adults; thus this task is reflective of naturally occurring environmental events. The task is also appropriate for children, virtually all of whom have been passengers in cars, and who are likely to perceive driving as an adult — and thus an attractive-activity.

The purpose of the present report is to provide initial data regarding the possible merits of including this task in future laboratory-based psychological stressor research by addressing four questions: (1) What amount of hemodynamic responsivity is elicited by the task? (2) What is the test-retest reliability of hemodynamic responses to the task? (3) How realistic does the task seem and how well does it elicit a variety of psychological states, such as nervousness, excitement, and involvement? (4) Are there gender differences in hemodynamic responses to the task?

\section{METHOD}

\section{Subjects}

Subjects were 11 White male medical students 23-28 years of age ( $M=24.2 \pm 1.5$ years) and 6 White and 1 African-American female graduate students $21-31$ years of age $(M=24.9 \pm 4.1$ years $)$. All subjects were paid $\$ 10$ for their participation. The fastest male and female drivers received an additional $\$ 25$.

\section{Apparatus}

A Panasonic Real 3DO Interactive Multi-player (Model FZ-1, Matsushita Electric Corporation of American, Secaucus, NJ) interfaced with a Kaiser Electro-Optics Vision Immersion headset (Model 500, Kaiser Aerospace and Electronics Company, Carlsbad, CA) was used to present the car-driving simulation task "Need for Speed" (Pioneer Productions and Electronic Arts, Inc., Canada).

The VIM-500 head-mount display weighs only 24 ounces, is eyeglasses compatible, is fully adjustable for each given head size and interpupillary distance, and has full-color multiple active matrix LCDs containing 360,000 color elements. The Panasonic 3DO system incorporates a handheld control pad. On the left-hand side is a circular directional pad. When pressed by the subject's left index finger, the car is steered to the left; likewise, pressure applied to the right-hand side of the directional pad steers the car to the right. Acceleration and braking are controlled by two buttons located on the right side of the control pad. These two buttons are operated by the subject's right index finger. Gear changing was programmed to be performed automatically for the task.

\section{Description of the Need for Speed Task}

Need for Speed immerses the subject in the cockpit of a car in which he/she has control over the steering, acceleration, and braking while driving in pursuit of another car. Various options are available with regard to type of car driven. In the present experiment, subjects drove a Porsche 911 in pursuit of a Lamborghini. Three options are provided with regard to the road terrain and conditions (inner city, coastal highway, and mountainous regions). We selected the coastal highway condition for our subjects. In this option, subjects drive along a road with hills on their left and an ocean on their right. This route provides a combination of stretches of level straight road, curves, blind hills, and corners, combined with twoway traffic. This condition is regarded by the manufacturer of the game as the middle level of difficulty from among the three conditions.

The main goal of the task is to complete the course as quickly as possible and beat the opponent to the finish line. The subject must first keep the car on the road: A crash occurs if the subject's car veers off on either side. When attempting to pass slower cars, the subject must avoid intermittent oncoming traffic. Again, crashes are possible; crashes can result in time delays, and three major crashes result in destruction of the car, which automatically ends the game. The second impediment to driving as fast as possible is the presence of police along the route. A radar detector is present in the cockpit, and if this is successfully monitored, police can be avoided altogether. If a siren is heard, the subject has two options. The first is to brake immediately, in which case the police officer gives a warning leading to a $3-\mathrm{sec}$ delay. The second is to try to outrun the police car. If caught, the subject receives a speeding ticket, which leads to a 5-sec delay. If two speeding tickets are issued, the subject is arrested by the officer and is eliminated from the game.

It should be noted that throughout the game, the subject receives appropriate visual and auditory stimuli in real time. For example, if the subject attempts to turn too quickly, the sound of tires screeching is heard while the visual feedback is commensurate with a careening car.

The game normally ends when the subject reaches the finish line, has three major crashes, or receives two speeding tickets. For the purposes of this study, the task was ended after 10 min of driving. All subjects completed the task.

\section{Hemodynamic Monitoring Equipment}

The hemodynamic parameters evaluated were systolic blood pressure, diastolic blood pressure, cardiac output, and calculated total peripheral resistance. Cardiac output was measured with a noninvasive thoracic electrical bioimpedance monitor (NCCOM-3 Model 6, Bo-Med Medical Manufacturing, Ltd., Irvine, CA). Two sets of eight silver/silver chloride spot electrodes (one current emitting, one sensing) were attached $5 \mathrm{~cm}$ apart at the level of the xiphoid notch in the midaxillary line and at the angle of the jaw on each side of the neck and connected to a set of leads interfaced with the NCCOM-3. We and other researchers have validated the NCCOM-3 via significant correlations between NCCOM-3 readings of and simultaneous thermodilution-derived estimates of cardiac output.

Blood pressure was evaluated with a Dinamap Adult/ Pediatric Vital Signs Monitor (Critikon Model 1846SX, Critikon, Inc., Tampa, FL) using an appropriately sized 
Table 1

Mean Baseline, Task Levels, Reactivity Scores, and Standard Deviations for Each Hemodynamic Parameter for Both Test Sessions

\begin{tabular}{|c|c|c|c|c|c|c|c|c|c|c|c|c|}
\hline \multirow[b]{3}{*}{ Parameter } & \multicolumn{6}{|c|}{ Session 1} & \multicolumn{6}{|c|}{ Session 2} \\
\hline & \multicolumn{2}{|c|}{ Baseline } & \multicolumn{2}{|c|}{ Task Level } & \multicolumn{2}{|c|}{ Reactivity Score } & \multicolumn{2}{|c|}{ Baseline } & \multicolumn{2}{|c|}{ Task Level } & \multicolumn{2}{|c|}{ Reactivity Score } \\
\hline & $M$ & $S D$ & $M$ & $S D$ & $M$ & $S D$ & $M$ & $S D$ & $M$ & $S D$ & $M$ & $S D$ \\
\hline $\mathrm{SBP}(\mathrm{mmHg})$ & 117.3 & 16.9 & 129.0 & 20.1 & 11.8 & 6.8 & 119.4 & 14.7 & 129.1 & 21.1 & 9.7 & 10.0 \\
\hline $\mathrm{DBP}(\mathrm{mmHg})$ & 64.9 & 9.8 & 71.3 & 12.1 & 6.4 & 6.1 & 63.6 & 8.5 & 71.5 & 11.8 & 7.9 & 5.5 \\
\hline $\mathrm{CO}(1 / \mathrm{min})$ & 5.8 & 1.3 & 6.0 & 1.4 & 0.1 & 1.1 & 5.7 & 1.0 & 6.3 & 1.3 & 0.6 & 0.67 \\
\hline $\mathrm{TPR}(\mathrm{mmHg} / \mathrm{L} / \mathrm{min})$ & 14.7 & 3.6 & 16.1 & 4.7 & 1.3 & 2.5 & 15.0 & 3.9 & 15.3 & 4.8 & 0.3 & 1.9 \\
\hline
\end{tabular}

Note-SBP, systolic blood pressure; DBP, diastolic blood pressure; CO, cardiac output; TPR, total peripheral resistance.

blood pressure cuff. The Dinamap has been validated for use during both rest and reactivity evaluations (Rosner et al., 1990). Cardiac output was calculated by the NCCOM-3 every successive 12 heart beats while the Dinamap was inflating and calculating blood pressures. Total peripheral resistance was derived by using concurrently measured blood pressure and cardiac output values (total peripheral resistance $=$ [systolic blood pressure $+2 \times$ diastolic blood pressure] $/ 3 /$ cardiac output) .

\section{Protocol}

After informed consent was obtained, subjects were taken to the laboratory for application of electrodes and blood pressure cuff. Since procedural variations can affect reliability (Saab et al., 1992), the two evaluations, which were separated by an average of $3.7 \pm 1.3$ weeks, were made as similar as possible in terms of time of day of evaluation and in terms of application of electrodes and stressor procedures. Subjects abstained from intake of caffeine for $4 \mathrm{~h}$ prior to the visit and of nonprescriptive medication use for $24 \mathrm{~h}$. The task protocol consisted of an initial 5 -min resting baseline followed by participation in the 10-min car-driving simulation task. Subjects were told that if they felt any physical discomfort, they should terminate the experiment immediately. No such problems were reported. All four hemodynamic parameters were assessed every other minute during the baseline period (Minutes 1, 3, and 5) and the task (Minutes $1,3,5,7$, and 9 ). Values obtained were subsequently averaged. Reactivity scores were calculated as mean task values minus mean baseline values.

\section{RESULTS}

Table 1 presents, for both testing sessions, the mean baseline and task levels for each of the hemodynamic parameters of interest. The first question, regarding amount of hemodynamic responsivity elicited by the task, was initially addressed via a multivariate repeated measures 2 (gender) $\times 2$ (phase; baseline vs. task level) $\times 2$ (time) analysis of variance (ANOVA) using the mean baseline and task levels as one repeated measure and the two evaluations as a second repeated measure. Dependent measures for the multivariate analysis were systolic and diastolic blood pressure, cardiac output, and total peripheral resistance. Significant main effects were observed for phase [baseline vs. task level; $F(1,16)=34.98, p<.001$ ] and gender $[F(1,16)=7.54, p<.02]$. The time effect was not statistically significant, nor were any of the interactions (all $p s>.15$ ). The lack of a time effect indicates that the patterns of mean hemodynamic responsivity did not differ significantly between sessions. Given these findings, the two baseline means were averaged, as were the two task-level means for each dependent variable. Subsequent univariate analyses were conducted using these averaged values separately for each dependent variable and task and gender as factors. Gender was found to be significant only for systolic pressure $(p<.001)$. Therefore, gender was dropped from the models for the remaining three hemodynamic parameters, and one-tailed paired $t$ tests were conducted. Collectively, the results indicated statistically significant increases from baseline on all four parameters (all $p s<.05$ ).

The second question, regarding degree of test-retest reliability, was addressed via Pearson product-moment correlation coefficients (Table 2). For reactivity scores, all coefficients were significant at least at the $p<.05$ level, whereas for task levels, all parameters were significant at $p<.001$.

The third question, regarding the degree of realism, excitement, nervousness, and involvement produced by the task, was assessed using the subject's self-report data. On the basis of a 5-point Likert scale response $(1=$ not at all; $5=$ very much), subjects reported levels of realism $(M=3.50 \pm 1.10)$, involvement $(M=3.4 \pm .98)$, excitement $(M=3.4 \pm .85)$, and nervousness $(M=2.4 \pm .92)$ that equated to average responses falling between moderate and very much. There were no significant gender differences on any of the self-ratings (all $p \mathrm{~s}>.14$ ).

The fourth question addressed the issue of possible gender differences in cardiovascular reactivity. As noted, the only significant difference observed involved systolic blood pressure, for which males exhibited greater systolic pressure at baseline and task level $[F(1,16)=$ $16.9, p<.001]$.

\section{DISCUSSION}

The purpose of this experiment was to provide an evaluation of the possible merits of utilizing a commercially available virtual reality car-driving task in cardiovascular reactivity research. Four questions were addressed-the amount and temporal stability of hemodynamic arousal caused by the car-driving stressor, the impact of the task 
Table 2

Pearson Product-Moment Correlation Coefficients $(n=18)$ for Intersession Test-Retest Reliability for Both Mean Task Levels and Mean Reactivity Scores

\begin{tabular}{ccc}
\hline & \multicolumn{2}{c}{ Correlation Coefficient } \\
\cline { 2 - 3 } Parameter & Task Level & Reactivity Score \\
\hline SBP $\dagger$ & $0.92 \dagger$ & $0.72 \dagger$ \\
$\mathrm{DBP} \dagger$ & $0.93 \dagger$ & $0.71 \dagger$ \\
$\mathrm{CO}^{*}$ & $0.82 \dagger$ & $0.54^{*}$ \\
$\mathrm{TPR}^{*}$ & $0.81 \dagger$ & $0.54^{*}$ \\
\hline
\end{tabular}

Note-SBP, systolic blood pressure; DBP, diastolic blood pressure; $\mathrm{CO}$, cardiac output; TPR, total peripheral resistance. ${ }^{*} p<.05 . \quad{ }^{\dagger} p<.001$.

on subjects' psychological reactions, and whether there were gender differences in hemodynamic reactivity.

Sizable increases in systolic and diastolic pressure were elicited that were attributed to slight increases in both peripheral resistance and cardiac output. Overall, these increases are very much in line with those reported using other psychological stressors for subjects of a similar age. For example, other video games and reaction time tasks have been shown to increase systolic and/or diastolic pressure from 6 to $18 \mathrm{mmHg}$, which is quite comparable to the present findings (see Turner, 1994). Examination of cardiovascular responses by gender revealed that males exhibited greater absolute levels of and greater increases in systolic pressure on both occasions. Other studies have shown that males often exhibit greater hemodynamic reactivity to tasks characterized by high levels of competitiveness (Matthews et al., 1986).

For a task to be considered successful within the context of identifying individuals predisposed to show a particular pattern of cardiovascular activation (a pattern that may be informative in predicting those at risk for later cardiovascular pathophysiology), that task must elicit similar responses from individuals across time. That is, hemodynamic arousal during the task must display significant test-retest reliability. In the present experiment, the mean hemodynamic task levels and mean reactivity change scores were comparable on the two occasions. Also, the test-retest reliability coefficients attained statistical significance at least at the $5 \%$ level. The observation of higher coefficients for task levels versus change scores is consistent with the literature (e.g., Turner \& Sherwood, 1991), and the range of correlation coefficients is consistent with the other findings for periods of comparable duration (Turner, 1994). Since statistical significance is more difficult to obtain with small samples, these findings provide clear evidence of the temporal stability of hemodynamic responses to the car-driving simulation stressor.

In summary, the development of a reliable battery of behavioral tasks for identifying risk for future cardiovascular pathophysiology may be of considerable use in cardiovascular behavioral medicine (Kamarck et al., 1992; Manuck, 1994). The evidence provided here strongly suggests that this virtual reality car-driving simulation stressor may be a useful addition to risk identification protocols. Cross-validation of these findings with larger sample sizes and samples that are more diverse with regard to age, ethnicity, and years of actual driving is needed. Whether exaggerated hemodynamic responsivity to this task is predictive of future cardiovascular disease remains to be determined. However, continued advancements in computer-aided design and software systems will no doubt result in development of other virtual reality simulations of real-life situations that may prove quite useful in the development of a battery of laboratory-based valid stressor tasks.

\section{REFERENCES}

Brod, J., Fencl, V., Hejl, Z, \& Jirka, J. (1959). Circulatory changes underlying blood pressure elevation during acute emotional stress (mental arithmetic) in normotensive and hypertensive subjects. Clinical Science, 18, 269-279.

Durel, L. A., Kus, L. A., Anderson, N. B., McNeilly, M., Llabre, M. M., Spitzer, S., SaAb, P. G., Efland, J., Williams, R., \& SChNeIderman, N. (1993). Patterns and stability of cardiovascular responses to variations of the cold pressor test. Psychophysiology, 30, $39-46$.

Kamarck, T. W., Jennings, J. R., Debski, T. T., Glickman-Weiss, E., JoHnSON, P. S., EDDY, M. J., \& MANUCK, S. B. (1992). Reliable measures of behaviorally-evoked cardiovascular reactivity from a PCbased test battery: Results from student and community samples. Psychophysiology, 29, 17-28.

MANUCK, S. B. (1994). Cardiovascular reactivity in cardiovascular disease: "Once more unto the breach." International Journal of Behavioral Medicine, 1, 4-31.

Matthews, K. A., Weiss, S. M., Detre, T., Dembroski, T. M., Falkner, B., MANUCK, S. B., \& Williams, R. B., JR. (1986). Handbook of stress, reactivity, and cardiovascular disease. New York: Wiley.

Moshell, J. M., \& DUNN-RoberTS, R. (1993). Virtual environments: Research in North America. In J. Thompson, (Ed.), Virtual reality: An international directory of research projects (pp. 3-26). Westport, CT: Meckler.

OBRIST, P. A. (1976). The cardiovascular-behavioral interaction: As it appears today. Psychophysiology, 13, 95-107.

OBRIST, P. A. (1981). Cardiovascular psychophysiology: A perspective. New York: Plenum.

Rosner, B. A., APPel, L. J., Raczynzki, J. M., Hebert, P. R., Whelton, P. K., Murphy, J. K., Miller, S. T., \& Oberman, A. (1990). A comparison of two automated monitors in the measurement of blood pressure reactivity. Annals of Epidemiology, 1, 57-69.

SaAB, P. G., Llabre, M. M., Hurwitz, B. E., Frame, C. A., Reineke, K. J., Fins, A. I., MCCAlla, J., CiePly, L. K., \& Schneiderman, N. (1992). Myocardial and peripheral vascular responses to behavioral challenges and their stability in Black and White Americans. Psychophysiology, 29, 384-397.

Saab, P. G., Matthews, K. A., Stoney, C. M., \& McDonald, R. H. (1989). Premenopausal and postmenopausal women differ in their cardiovascular and neuroendocrine responses to behavioral stressors. Psychophysiology, 26, 270-280.

TURNER, J. R. (1994). Cardiovascular reactivity and stress: Patterns of physiological response. New York: Plenum.

Turner, J. R., Carroll, D., \& Courtney, H. (1983). Cardiac and metabolic responses to "space invaders": An instance of metabolicallyexaggerated cardiac adjustment? Psychophysiology, 20, 544-549.

TURNER, J. R., \& SHERwOOD, A. (1991). Postural effects on blood pressure reactivity: Implications for studies of laboratory-field generalization. Journal of Psychosomatic Research, 35, 289-295.

(Manuscript received January 17, 1996; revision accepted for publication June 5, 1996.) 